\title{
Video Article \\ Improving High Viscosity Extrusion of Microcrystals for Time-resolved Serial Femtosecond Crystallography at X-ray Lasers
}

\author{
Daniel James ${ }^{1}$, Tobias Weinert ${ }^{1}$, Petr Skopintsev ${ }^{1}$, Antonia Furrer ${ }^{1}$, Dardan Gashi ${ }^{1,2}$, Tomoyuki Tanaka ${ }^{3,4}$, Eriko Nango ${ }^{3,4}$, Przemyslaw Nogly ${ }^{1,5}$, \\ Joerg Standfuss ${ }^{1}$ \\ ${ }^{1}$ Division of Biology and Chemistry - Laboratory for Biomolecular Research, Paul Scherrer Institut \\ ${ }^{2}$ Photon Science Division - SwissFEL, Paul Scherrer Institut \\ ${ }^{3}$ RIKEN SPring-8 Center \\ ${ }^{4}$ Department of Cell Biology, Graduate School of Medicine, Kyoto University \\ ${ }^{5}$ Department of Biology, ETH Zürich
}

Correspondence to: Joerg Standfuss at joerg.standfuss@psi.ch

URL: https://www.jove.com/video/59087

DOI: doi:10.3791/59087

Keywords: Chemistry, Issue 144, time-resolved serial crystallography, lipidic cubic phase, bacteriorhodopsin, membrane proteins, high viscosity injector, three-way coupler, X-ray free eletron laser

Date Published: 2/28/2019

Citation: James, D., Weinert, T., Skopintsev, P., Furrer, A., Gashi, D., Tanaka, T., Nango, E., Nogly, P., Standfuss, J. Improving High Viscosity Extrusion of Microcrystals for Time-resolved Serial Femtosecond Crystallography at X-ray Lasers. J. Vis. Exp. (144), e59087, doi:10.3791/59087 (2019).

\section{Abstract}

High-viscosity micro-extrusion injectors have dramatically reduced sample consumption in serial femtosecond crystallographic experiments (SFX) at X-ray free electron lasers (XFELs). A series of experiments using the light-driven proton pump bacteriorhodopsin have further established these injectors as a preferred option to deliver crystals for time-resolved serial femtosecond crystallography (TR-SFX) to resolve structural changes of proteins after photoactivation. To obtain multiple structural snapshots of high quality, it is essential to collect large amounts of data and ensure clearance of crystals between every pump laser pulse. Here, we describe in detail how we optimized the extrusion of bacteriorhodopsin microcrystals for our recent TR-SFX experiments at the Linac Coherent Light Source (LCLS). The goal of the method is to optimize extrusion for a stable and continuous flow while maintaining a high density of crystals to increase the rate at which data can be collected in a TR-SFX experiment. We achieve this goal by preparing lipidic cubic phase with a homogenous distribution of crystals using a novel threeway syringe coupling device followed by adjusting the sample composition based on measurements of the extrusion stability taken with a highspeed camera setup. The methodology can be adapted to optimize the flow of other microcrystals. The setup will be available for users of the new Swiss Free Electron Laser facility.

\section{Video Link}

The video component of this article can be found at https://www.jove.com/video/59087/

\section{Introduction}

Serial femtosecond crystallography (SFX) is a structural biology technique that exploits the unique properties of X-ray free electron lasers (XFEL) to determine room temperature structures from thousands of micrometer-sized crystals while outrunning most of the radiation damage by the "diffraction before destruction" principle ${ }^{1,2,3}$.

In a time-resolved extension of SFX (TR-SFX), the femtosecond pulses from the XFEL are used to study structural changes in proteins ${ }^{4,5}$. The protein of interest is activated with an optical laser (or another activity trigger) just prior to being shot by the XFEL in a pump-probe setup. By precisely controlling the delay between pump and probe pulses, the target protein can be captured in different states. Molecular movies of structural changes over eleven orders of magnitude in time demonstrate the power of the new XFEL sources to study the dynamics of several protein targets $6,8,9,10,11,12,13$. Principally, the method joins the dynamic spectroscopic and static structural techniques into one, providing a glimpse into protein dynamics at near atomic resolution.

Simple systems for TR-SFX may contain an endogenous trigger of activation with a photo-sensitive component like retinal in bacteriorhodopsin $(\mathrm{bR})^{9,10}$, the chromophores in photosystem II ${ }^{12,13}$, photoactive yellow protein (PYP) $)^{6,7}$ reversibly photoswitchable fluorescent protein ${ }^{11}$, or a photolyzable carbon monoxide in myoglobin ${ }^{8}$. Exciting variations of the technique still in development rely on mix and inject schemes ${ }^{14,15}$ to study enzymatic reactions or an electric field used to induce structural changes ${ }^{16}$. Given that XFEL sources have only been available for a few years and extrapolating past successes into the future, the method shows potential as a real game-changer with respect to our understanding of how proteins function.

Because biological samples are destroyed by a single exposure to a high power XFEL pulse, new approaches to protein crystallography were necessary. Among these procedures, the ability to grow large amounts of uniform microcrystals needed to be developed ${ }^{17,18,19}$. To enable 
data collection at an XFEL, these crystals must be delivered, discarded, and then renewed for each XFEL pulse. Given that XFELs fire usable pulses at $10-120 \mathrm{~Hz}$, sample delivery must be fast, stable, and reliable, while also keeping the crystals intact and limiting consumption. Among the most successful solutions is a high viscosity micro-extrusion injector, which delivers a continiously streaming column of room temperature crystal-laden lipidic cubic phase (LCP) across the pulsed X-ray beam ${ }^{20}$. Randomly oriented crystals, embedded in the LCP stream, that are intercepted by the XFEL pulses scatter X-rays onto a detector where a diffraction pattern is recorded. LCP was a natural choice for a sample delivery medium as it is frequently used as a growth medium for membrane protein crystals ${ }^{17,21,22,23}$, yet other high viscosity carrier media $^{24,25,26,27,28,29,30}$ and soluble proteins ${ }^{31}$ have also been used in the injector. SFX with the high viscosity injector has been successful during the structure determination of membrane proteins ${ }^{13,32}$ including G protein-coupled receptors (GPCRs) ${ }^{33,34,35,36,37}$, with data quality sufficient for native phasing ${ }^{38,39}$ while being both time and sample efficient. Currently, these injectors are being used more routinely for room temperature measurements at synchrotron sources ${ }^{28,30,40,41}$ as well as during the more technically demanding TR-SFX experiments at XFELs ${ }^{9,10,13,42}$.

Comparable TR-SFX experiments have been carried out using other injector types like liquid phase delivery in a flow focused nozzle ${ }^{6,7,12}$, however, this method requires protein amounts not available for many biologically interesting targets. For the determination of static structures using viscous extrusion an average consumption of $0.072 \mathrm{mg}$ of protein per 10,000 indexed diffraction patterns in comparison to $9.35 \mathrm{mg}$ for the liquid jet nozzles have been reported (i.e., about 130 times more sample efficient) ${ }^{20}$. The high viscosity injector has been shown to be a viable sample delivery device for TR-SFX while only sacrificing some of this sample efficiency ${ }^{43}$. In Nogly et al. $(2018)^{10}$, for example, sample consumption was about $1.5 \mathrm{mg}$ per 10,000 indexed patterns, which compares favorably to similar TR-SFX experiments using the PYP where average sample consumption was much higher with $74 \mathrm{mg}$ of protein per 10,000 indexed patterns ${ }^{6}$. High viscosity injectors thus have clear advantages when the amount of protein available is limiting or when crystals are grown directly in LCP.

For TR-SFX using high viscosity injectors to yield the most reliable data several technical issues need to be addressed: the flow speed needs to remain above a minimum critical value; the hit-rate should be maintained at a level that does not render data collection slow (e.g., greater than 5\%); and sample has to be delivered without excessive disruptions. Ideally, these conditions are already met long before a scheduled TRSFX experiment to use available XFEL time as efficiently as possible. Pricipally, a slowdown in the LCP stream may allow probing crystals that were activated with more than one optical laser pulse and result in mixed active states, or probing pumped material when umpumped material is expected in the beam. An additional benefit of injection pre-testing is that downtime during data collection at an XFEL is minimized as time relegated to replacing clogged nozzles, changing out non-extruding samples, and other maintenance tasks is reduced.

Here, we present a method to optimize sample delivery for TR-SFX data collection with a high viscosity micro-extrusion injector. For simplicity, the described methods do not rely on access to an X-ray source, although work at a synchrotron beamline ${ }^{29}$ would provide further information on expected hit rates and crystal diffraction. Our protocols were developed to optimize experiments to capture retinal isomerization in the protonpump bacteriorhodopsin ${ }^{10}$ and are carried out in two phases starting with preparing crystal samples for extrusion followed by monitoring the extrusion using a high-speed camera setup. In phase one, the crystal-laden LCP is mixed with additional LCP, low transition temperature lipids, or other additives to ensure the final mixture is suitable for delivery into the sample environment without clogging or slowing. A new threeway syringe coupler was developed to improve mixing performance and sample homogeneity. The second phase consists of an extrusion test recorded by a high-speed camera to directly measure the extrusion speed stability. Following the analysis of the video data, adjustments can be made to the sample preparation protocol to improve experimental outcomes. These procedures can be adapted to prepare other proteins for TR-SFX data collection, with minimal modifications, and will contribute to the efficient use of limited XFEL beamtime. With new XFEL facilities just starting their operation ${ }^{44,45}$ and the transfer of injector-based serial data collection methods to synchrotrons ${ }^{28,30,40,41}$, the next few years will surely continue to provide exciting new insights into the structural dynamics of an ever-wider range of protein targets.

\section{Protein Crystal Sample Preparation}

1. About 30 min before the sample is to be injected, load $50 \mu \mathrm{L}$ of crystal-laden monoolein based LCP in a $100 \mu \mathrm{L}$ syringe.

2. For injection at atmospheric pressure: Load $10 \mu \mathrm{L}$ of liquid paraffin into the back of a second syringe. Holding the syringe vertically, expel the air bubbles from the syringe.

1. For injection into vacuum environment: Load $5 \mu \mathrm{L}$ of MAG 7.9 and $5 \mu \mathrm{L}$ of liquid paraffin into the back of a second syringe. Holding the syringe vertically, expel the air bubbles from the syringe.

3. Connect the syringe with paraffin/MAG 7.9 to a standard syringe coupler, purge the air from the coupler by gently pressing on the plunger until a small volume $(<1 \mu \mathrm{L})$ of paraffin/lipid is visible at the tip of the coupler needle.

4. Connect the sample syringe to the syringe coupler, taking care to not introduce any air into the sample. Mix in the lipid/paraffin by passing the sample material through the coupler multiple times.

5. Load $20 \mu \mathrm{L}$ of premixed LCP $(27 \%$ PEG, $100 \mathrm{mM}$ Sorensen $\mathrm{pH} 5.6+\mathrm{MO})$ into another $100 \mu \mathrm{L}$ syringe. Remove air bubbles as necessary.

6. Remove the empty syringe from the coupler and attach the premixed LCP to the crystal containing syringe using a standard syringe coupler. Pass the sample through the coupler 100 times.

NOTE: Sample mixing may heat the sample slightly ${ }^{46}$. Mixing should be done at a slow rate where the temperature of the sample can be held reasonably constant.

7. Inspect the sample for transparency against a source of light. If a clear homogeneous LCP has formed, go to step 1.9.

8. To bring the sample into the cubic phase, add $3 \mu \mathrm{L}$ of monoolein and mix 50 times (as described above). Repeat this procedure just until a transparent phase has formed to avoid an excess of monoolein.

NOTE: The formation of the LCP is temperature dependent ${ }^{47}$ and best results are achieved slightly above $20{ }^{\circ} \mathrm{C}$. The amount of additional monoolein needed will depend on the volume of residual precipitant solution carried over from crystallization.

9. As a preliminary test for sample stiffness (as expected from the LCP phase) and extrude-ability, detach the empty syringe from the syringe coupler and, holding the syringe vertically, squeeze a small amount of sample $(<2 \mu \mathrm{L})$ through the coupler. If the extruded sample forms an upright cylinder, then the sample is ready for extrusion testing.

10. Adjust the total volume of the sample to $100 \mu \mathrm{L}$ by adding more premixed LCP (as in step 1.5). 
11. Attach the sample syringe and two empty syringes to the three-way syringe coupler (purging the air from the coupler as before). Mix at least 50 times (or until homogeneous) by passing half of the sample into the second syringe and then pressing both halves of the sample into the third syringe simultaneously.

12. Place the syringe containing the mixed sample under a stereo microscope to verify a homogenous distribution of crystals.

\section{Testing Sample Extrusion Using a High-Speed Camera Setup}

1. Determining experimental parameters.

1. Select the nozzle size for the test.

NOTE: Nozzle sizes are typically 50 or $75 \mu \mathrm{m}$ internal diameter (ID), but any size from roughly 30-100 $\mu \mathrm{m}$ ID may be considered.

Selection is based on a balance between mean clogging time, background scattering, sample consumption, and hit-rate.

2. Calculate optimum flow speed based on experimental laser spot size (1/e diameter), and the data collection scheme (e.g., interleaved light and dark) that will be used at the XFEL.

3. Calculate the desired flow rate $\left(Q_{s}\right)$ of the sample from the optimum flow speed $\left(v_{e}\right)$ and the selected nozzle diameter $(d)$ :

$Q_{s}=v_{e} \pi \frac{d^{2}}{4}$

Determine the flow rate $\left(Q_{P}\right)$ that should be entered at the HPLC pump based on the amplification factor $(A)$ of the injector.

$Q_{P}=Q_{s} A$

Calculate the maximum pressure $\left(P_{M A X}\right)$ from the rated pressure of the nozzle fittings $\left(R_{M A X}\right)$ and the amplification factor of the injector $(A)$ :

$P_{M A X}=\frac{R_{M A X}}{A}$

2. Setup of the high viscosity extrusion injector for offline use as shown in Figure 1.

NOTE: The injector functions by means of hydraulic extrusion powered by an HPLC pump, and uses a co-flowing helium gas sheath controlled by a gas regulator. The pump and regulator setup are not discussed in this protocol. See chapter four in James (2015) ${ }^{48}$ for a detailed manual.

1. Purge the pump and all water lines to ensure the flowrates are accurate. Purge the hydraulic stage of the injector.

2. Mount the injector (or the camera) on a three-axis stage to facilitate framing and focus for the high-speed videos. Leave space around the injection point for the objective lens, illumination, reflective screen, and a small beaker to catch the spent sample.

3. Construct a "dummy nozzle" (an empty reservoir with a nozzle attached) and install it on the injector to facilitate positioning, focus, and illumination.

4. Mount the high-speed camera with the nozzle tip near the focal point of the objective.

5. Position the reflective screen behind the injector and adjust the illumination to front-light the nozzle.

6. Turn on and connect to the camera with the supplied software. With a live video running for visual feedback, position the nozzle tip in the center of the frame, and bring it into focus with the three-axis stage.

7. Adjust the illumination until the nozzle is clearly visible and the background is evenly illuminated.

3. Configuring the camera to record high-speed time-lapse video.

1. Set framerate to $1000 \mathrm{fps}$. Set resolution to $512 \times 512$ pixels.

2. With the exposure time now set by the frame rate, adjust the illumination level until the nozzle is visible (i.e., not under- or overexposed). Reposition the nozzle tip so that it is centered left to right and is located in the top third of the frame.

3. Run any background correction or white balance operations.

4. Set up the camera in time-lapse mode. Set interval to $\mathbf{3 0} \mathrm{s}$ and repeats to $\mathbf{4 0}$ times, set the trigger mode to random (or random reset), and enter the number of frames to record to 1000.

4. Load the reservoir with $20 \mu \mathrm{L}$ of the test sample, and attach the capillary nozzle.

5. Attach the filled reservoir to the injector. Attach the gas line to the port on the nozzle and start the gas flow.

6. Manually advance the piston by opening the in-line valve and pressing the syringe. Close the valve when the piston makes solid contact with the sample in the reservoir.

7. Program the pump with the calculated flow rate and set the maximum pressure to the calculated value (see step 2.1.3).

8. Simultaneously start the pump and the camera recording.

9. As the extrusion begins, adjust the gas pressure to increase stability. Increase gas pressure if the fluid forms a drop at the tip of the nozzle instead of a column. Decrease pressure if the extrusion is broken due to excessive shear from the gas flow, or is oscillating rapidly (whipping).

10. Monitor the extrusion (10 min is usually enough to recognize a homogenous flow condition) and, when the pump pressure sharply ramps up near the expected end time, stop recording, shut off the pump, and vent the system pressure by opening the relief valve.

11. Analysis of the video files

NOTE: Sample extrusion that is plainly inadequate does not require a detailed analysis. However, it is still useful to identify the failure mode so that the sample may be optimized.

1. Open the video file with the analysis software (e.g., $\left.\mathrm{Fiji}^{49}\right)$ and calibrate the measurement tools.

2. Calibrate the measurements by selecting a line of known length in the video image with the line selection tool. Open the Set Scale window via Analyze | Set Scale and enter the known distance and the units of measurement. NOTE: The known diameter of the nozzle provides an adequate calibration length for these measurements.

3. Find a frame in the video where there is a trackable feature visible (e.g., a crystal) in the extrusion. Record the frame number.

4. Advance the video to a frame where the same feature is visible but has moved from its position in the previous step. Record the frame number.

5. Using the straight line measurement tool, measure the distance from the feature start and endpoint via Analyze | Measure. NOTE: The longer the trackable distance, the fewer errors in measurement will affect the calculations 
6. Calculate the jet speed from the measured distance and the elapsed time (the number of elapsed frames divided by framerate.)

7. Repeat steps 2.11.3-2.11.6 a few times for every segment of the video.

8. Plot the data series as in Figure 2.

\section{Representative Results}

The ideal starting material for the procedures described here (Figure 3 ) are high densities of microcrystals incorporated into viscous carrier medium for the injector. The procedure calls for about $50 \mu \mathrm{L}$ of crystal laden carrier for each preparation. These can be grown directly in LCP as with the $\mathrm{bR}^{9,10}$ used here, as an example (Figure 4), or prepared using crystals grown in conventional vapor diffusion setups. An excellent video guide to crystallization directly in gastight syringes can be found in Ishchenko et al. $(2016)^{37}$. With bR, the ideal diameter of the crystalline plates is around $20 \mu \mathrm{m}$ as a good compromise between diffraction power and homogenous activation by the pump laser. Protein crystals, prepared using the protocols as explained above, were used to collect TR-SFX data on the proton pump bR which revealed the ultrafast changes that occur after photon absorbtion (Figure 5).

After sample preparation using the three-way coupler (Figure 6A,B), visual inspection of the sample material in the syringe shows sample homogeneity (Figure 6C,D), and microscope images of the mixed sample, both in the syringe and on a slide, can confirm the density of the crystals and allows for measurements of crystal sizes, crystal size distribution, and crystal density. The sample is in the cubic phase when the delivery medium is clear (Figure 7) and viscous. Turbid mixtures are an indication that the sample is in a sponge phase or lamellar phase, but are not conclusive as high crystal density may obscure the LCPs clarity. A brief low-pressure test to identify the liquid sponge phase can be performed by pulling the syringe plunger away from the sample in the back of the syringe. Lamellar phase can be easily identified through its birefringence with polarized light microscopy. These tests coupled with a pre-extrusion test are usually sufficient to justify a test in the injector.

The high repetition rate of XFELs requires a flow speed that cannot be adequately observed with low frame rate cameras. Therefore, the extrusion characterization is done using a high-speed camera (coupled to a high magnification lens) that can record time-lapse video. Video data is high-speed in that frames are recorded at $1000 \mathrm{fps}$, and also time-lapse in that video is recorded for $1 \mathrm{~s}$ every $30 \mathrm{~s}$. This data collection scheme will allow for data collection during the entire sample test (about $10 \mathrm{~min}$ ) without creating unmanageable video files. Even with this reduced data set size, video files may still be in excess of $50 \mathrm{~GB}$. Care should be taken to ensure adequate storage is available to save video data before the test begins.

During the jet test (Figure 1), the sample should extrude a long (more than $200 \mu \mathrm{m}$ ) continuous column of LCP that moves at a nearly constant velocity (Figure 2). Samples that run in a dripping mode indicate that the sample viscosity is too low (Figure 2). Samples can often be recovered and mixed with more monoolein or paraffin to adapt viscosity. Samples that form a column should be tested with the high-speed camera. Data from the recording should show that the extrusion stays above a minimum speed dictated by your experimental parameters. Extrusion speed is determined by measuring the linear distance a feature in the LCP stream travels over a number of frames. Extrusion slow-downs that can be attributed to anomalous clogs warrant retesting. If they persist jetting should be improved by additives like paraffin, by reducing the density of crystals, or by selecting a larger diameter nozzle. Crystals not normally grown in a viscous medium (e.g., vapor diffusion) can be peletted by centrifugation and incorporated into LCP or other viscous carriers. For examples, see previous publications ${ }^{24,25,26,27,29,30}$. 


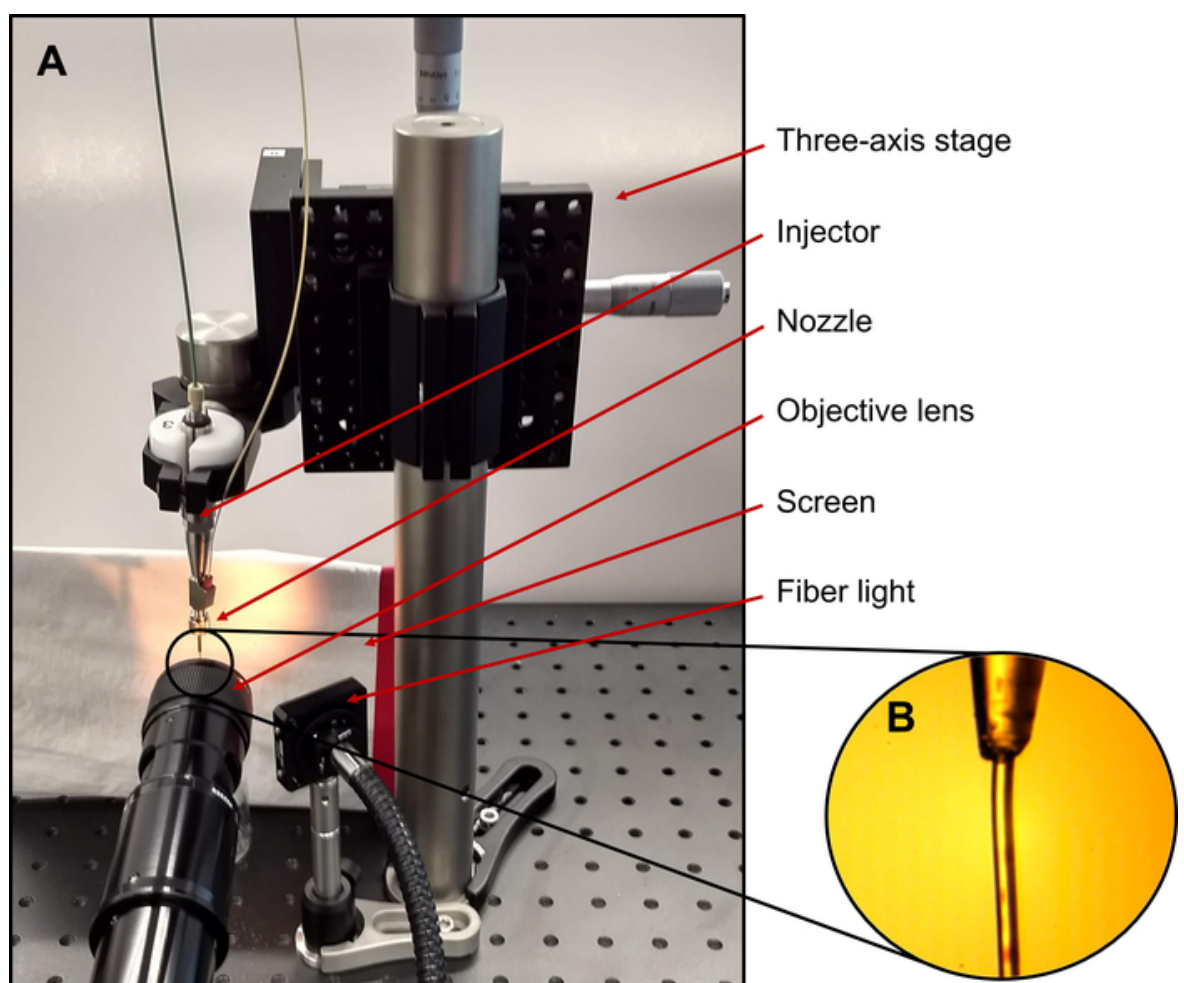

Figure 1: High-speed camera bench setup. A photograph of a typical benchtop setup with the essential parts labeled. The three-axis stage provides flexibility in framing and focusing the image. The injector (shown with the nozzle and reservoir attached) is mounted vertically, and directly in front of the objective lens. The illumination in this example is provided by a single fiber light illuminating the sample off axis and providing a bright field on the screen. A sample mounted and illuminated in this way provides an image like the one in inset B. Please click here to view a larger version of this figure. 

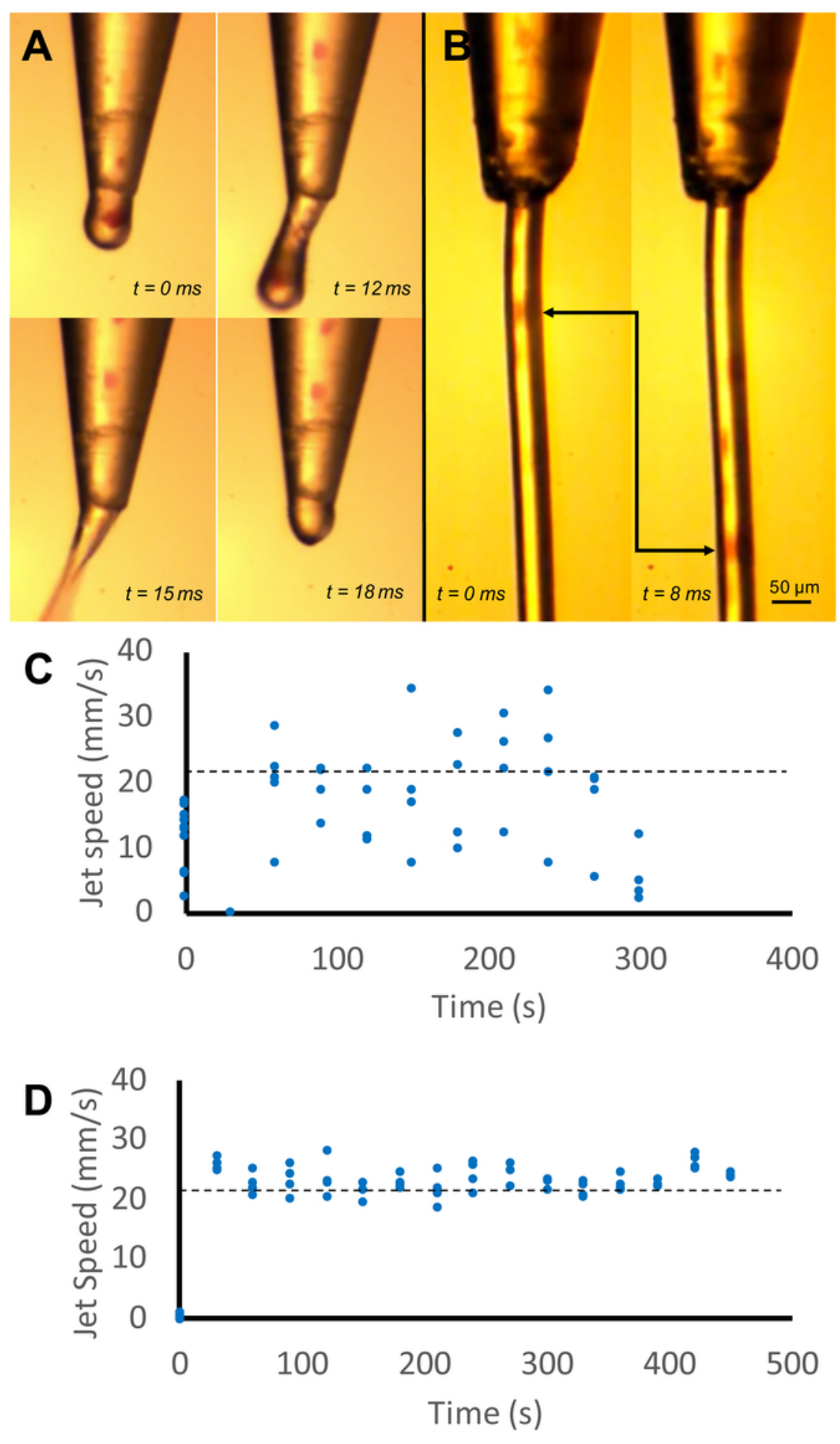

Figure 2: High-speed camera testing and data analysis. Pictured above are two LCP extrusion tests. Test A shows bR crystals in LCP extruding in a dripping mode indicating that the sample is not optimized. Test B shows LCP extrusion forming a continuous column of LCP where crystals can be tracked, and extrusion speed can be calculated. In this example, a crystal embedded in the LCP column (50 $\mu \mathrm{m}$ diameter) moves $301 \mu \mathrm{m}$ in $8 \mathrm{~ms}(37.6 \mathrm{~mm} / \mathrm{s})$. Two data sets from different preparations of bacteriorhodopsin crystals in LCP are shown. Four (or more) data points from each second of time-lapse high-speed camera video ( $1 \mathrm{~s}$ of recording every $30 \mathrm{~s}$ ) were plotted. A large spread between data points taken from a single second data collection period indicate short-term speed variation. While a moving average over the whole data series shows the longer fluctuations in the flow. The top series is from a poorly extruding sample (high chance for light contamination with two consecutive pump laser pulses), and the bottom series is from a sample that performed optimally. The horizontal dotted line indicates the target extrusion speed set via the HPLC pump. Please click here to view a larger version of this figure. 


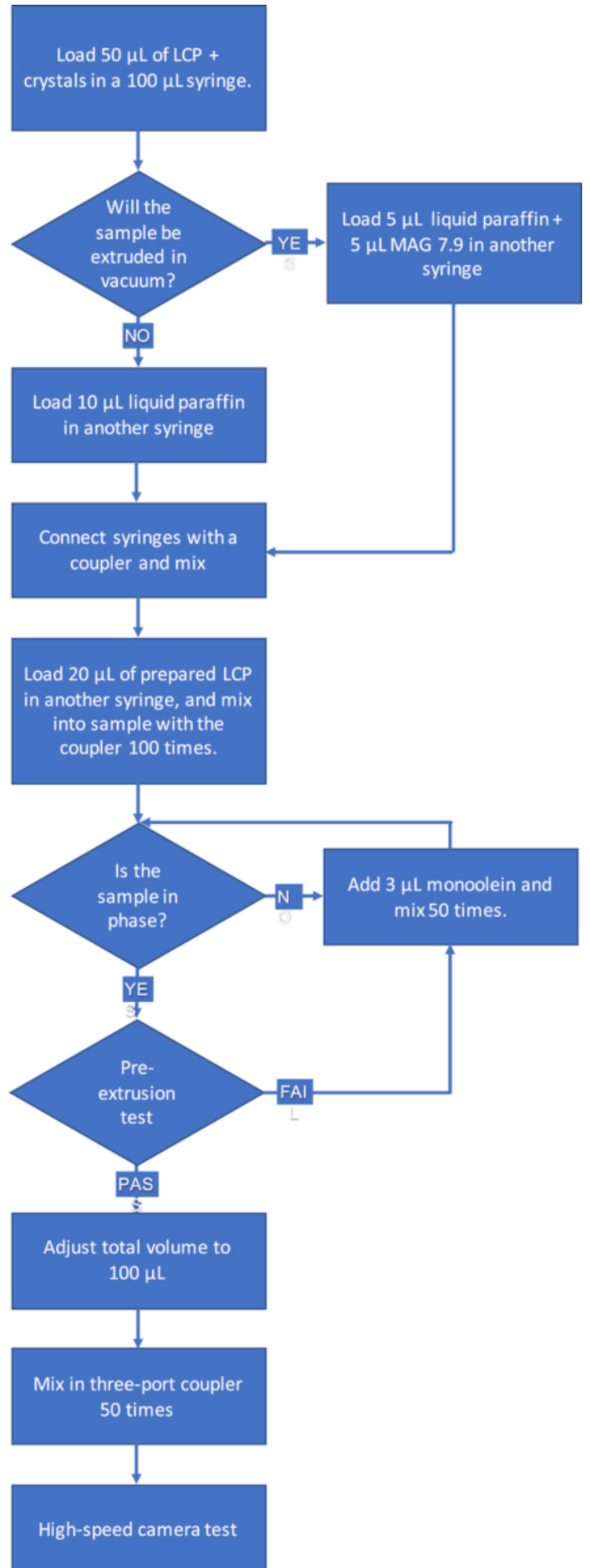

Figure 3: Sample preparation flowchart. Sample preparation starts with a high density of protein crystals embedded in LCP. The sample is then mixed with low transition temperature lipid, paraffin, and prepared LCP until the sample comes into the cubic phase, and passes a series of small tests to indicate that the sample will extrude in the injector. Please click here to view a larger version of this figure. 


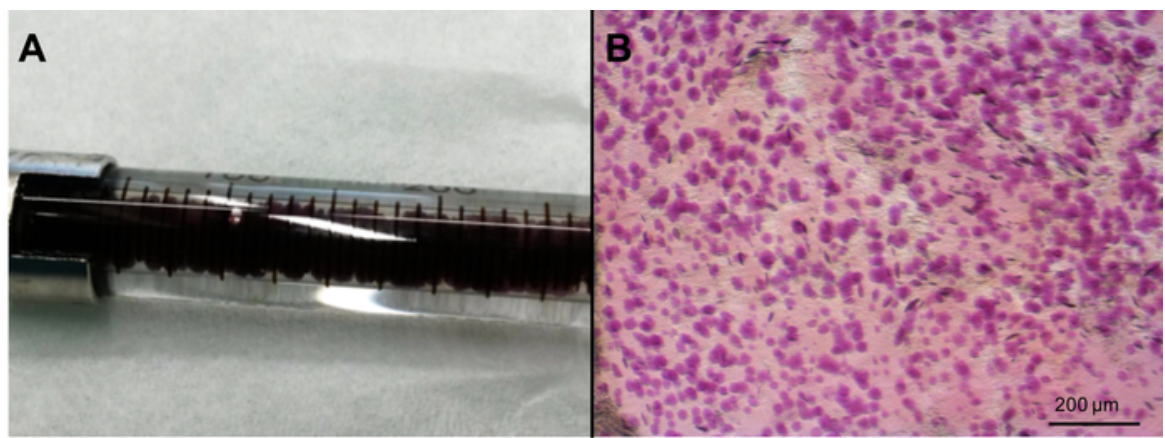

Figure 4: Bacteriorhodopsin crystals as starting material. Crystals are grown in gas-tight syringes $(A$,$) with LCP immersed in the precipitant$ solution. Crystallization is optimized for a high density of crystals with a possibly narrow size distribution (B). Please click here to view a larger version of this figure.

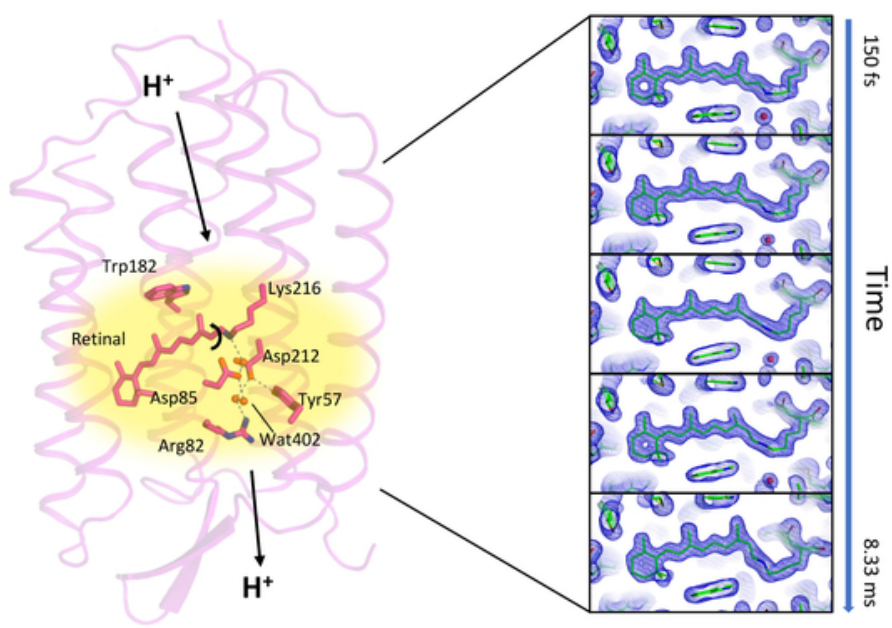

Figure 5: Structural changes in bacteriorhodopsin. TR-SFX reveals ultra-fast changes in the structure of the proton pump bacteriorhodopsin. Here is pictured a model of bR derived from TR-SFX data obtained from crystals prepared using the protocols outlined in this work. The left panel shows the model with highlighted features in the retinal binding pocket. The right panel shows the changes in the retinal from femtoseconds to milliseconds. This figure was reproduced from Nogly et al. $(2018)^{10}$ with permission. Please click here to view a larger version of this figure. 


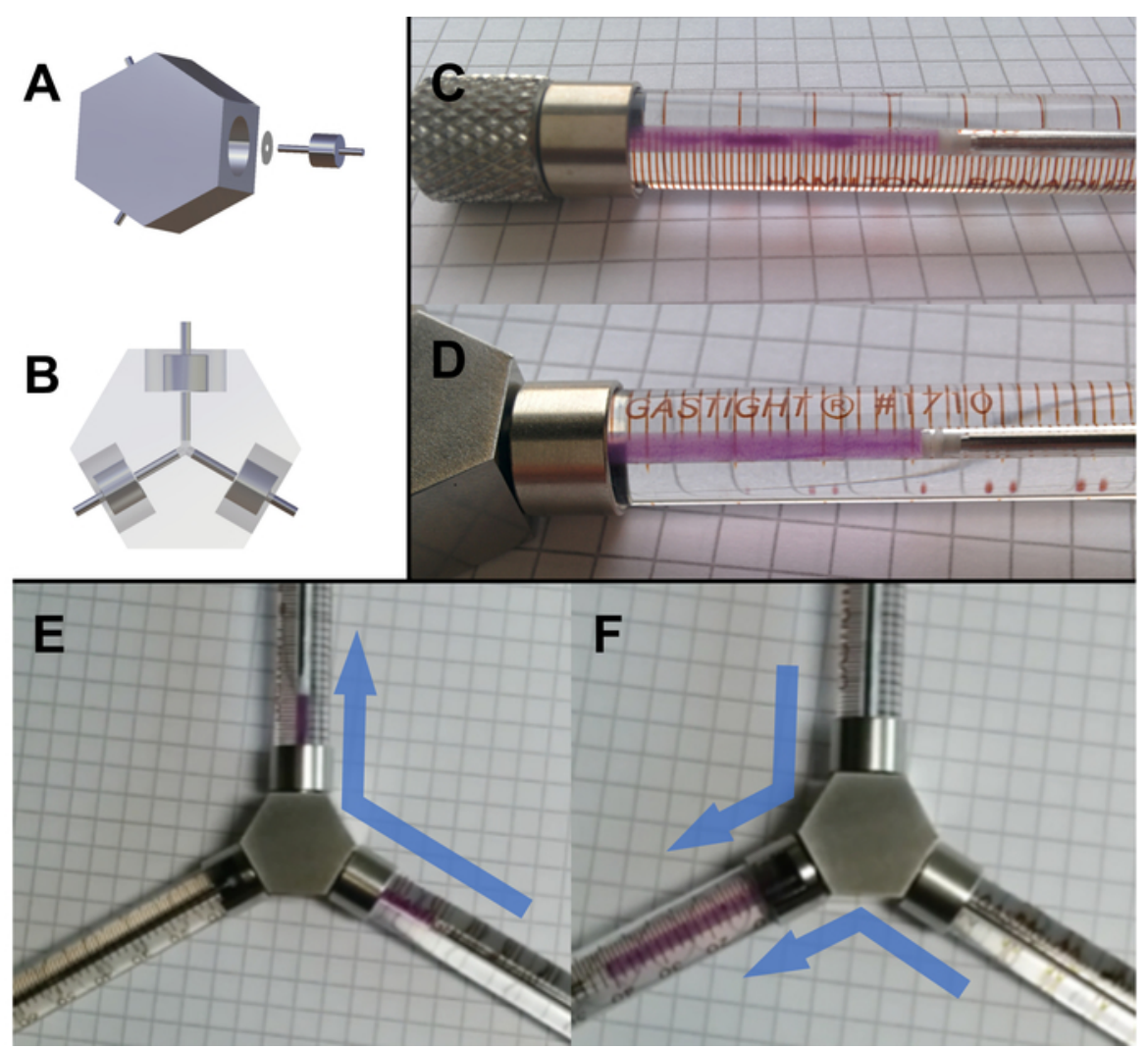

Figure 6: The three-way coupler. The three-way coupler is a reduced dead-volume $Y$ mixer designed for compatibility with gastight syringes. $3 \mathrm{D}$ renderings of the coupler, show an exploded view of the assembly $(A)$, and a transparent view showing the mixing channel $(B)$. The figure shows an inhomogeneous crystal distribution after mixing with the standard coupler $(C)$, and the resultant suspension after mixing in the threeway coupler (D). Mixing is accomplished by pushing roughly half of the sample into another syringe (E), and then pushing both halves into the remaining syringe together as illustrated by the blue arrows (F). Please click here to view a larger version of this figure. 


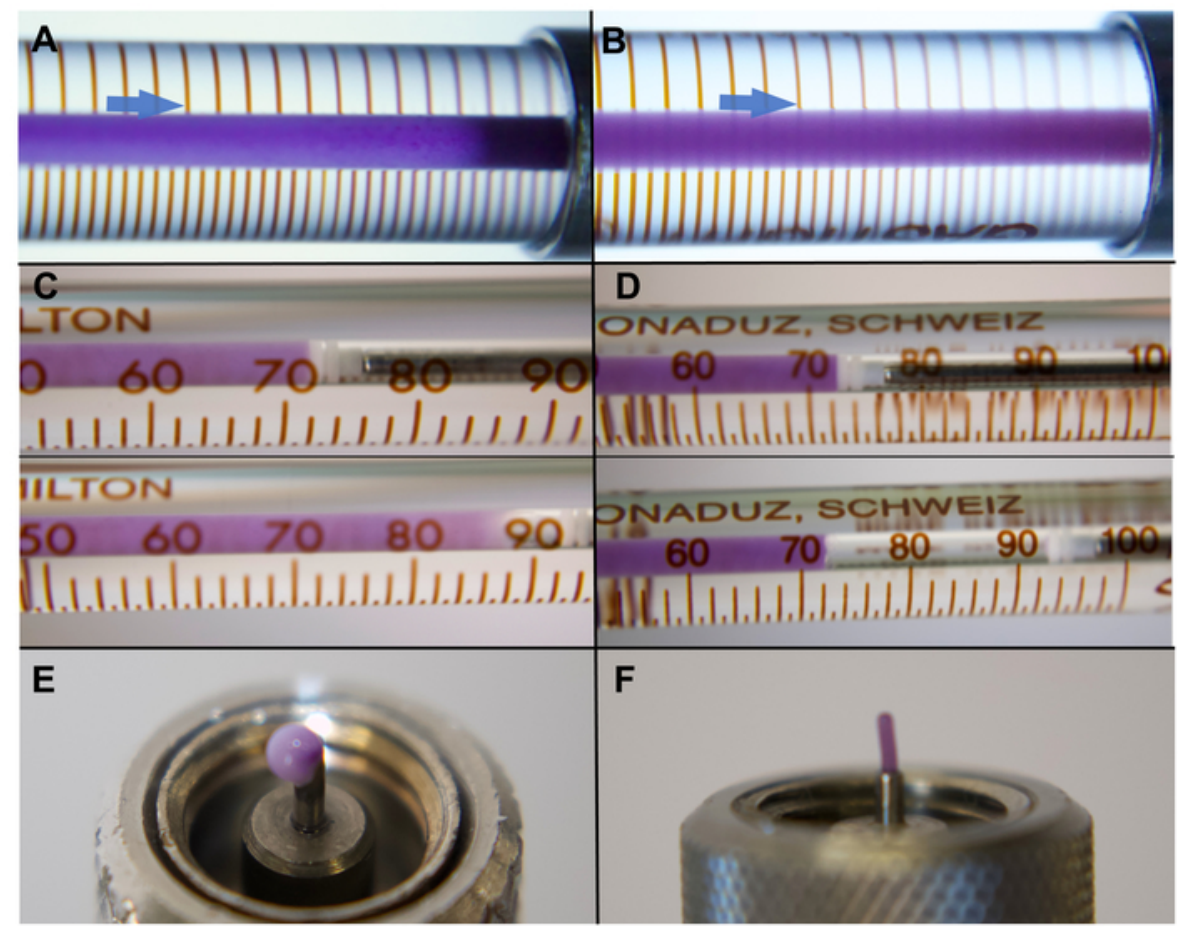

Figure 7: Pre-injection testing indicators. During sample preparation, several small tests are used to indicate that the sample is in phase, and adequately viscous. Optical clarity is one indicator that the sample is likely in the cubic phase. (A) A turbid sample is shown in the syringe. (B) A clear sample is shown, note the graduation lines visible through the sample despite the high density of micro-crystals. When the lipid matrix is in the cubic phase, it remains solid-like when under no stress. A low-pressure test is performed by pulling the syringe away from the back of the sample. When the sample is too liquid-like (indicative of the sponge phase) the sample expands to fill the volume (C). In a positive result of the same test (D), the sample remains static despite the withdrawn plunger. Finally, a macro-scale extrusion test is performed by pressing a small amount of sample through the syringe coupler. A droplet forming at the nozzle tip (E) or a quickly bending cylinder of LCP indicates a sample that is not viscous enough. If the sample extrudes, forms a cylinder, which remains upright over time (F), then the sample may advance to high-speed camera testing. Please click here to view a larger version of this figure.

\section{Discussion}

The TR-SFX method with the viscous extrusion injector has proven to be a viable technique for structural dynamics studies of

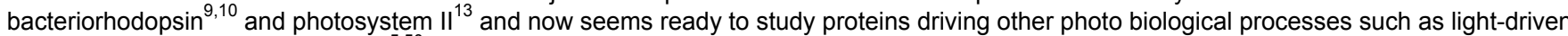
ion transport or sensory perception ${ }^{5,50}$. The protocols described above were designed to maximize the success of TR-SFX data collection on bacteriorhodopsin, yet we believe can act as a template to optimize data collection on other samples. In this way, much of the precious beamtime currently lost because of clogging or poor sample extrusion could instead be used to collect better data at more time delays.

The most difficult, and critical step in the protocol is the addition of small amounts of monoolein that brings the lipid matrix into the cubic phase (step 1.8). The difficulty lies in the subtlety of the phase transition and the negative effect on the sample when monoolein is added in excess.

Modifications to the methodology should be implemented to accommodate different protein crystals. These may not be compatible with the same additives or the amount or required mixing. On the other hand, breaking crystals into smaller pieces during the mixing process may not compromise diffraction quality and can even improve jetting for example when long needles break into shorter fragments. Different additives could be substituted in place of paraffin, which is added to improve flow speed stability 9 . MAG 7.9/9.7 is added to prevent the trasnistion to lamellar phase that may occur when injecting into vacuum environments ${ }^{20}$ (like the CXI endstation at LCLS), but may be omitted if the experiment is done at atmospheric pressure. In our experience, many crystals from soluble proteins can be stably incorporated into LCP, yet somewhat ironically membrane protein crystals not directly grown in lipidic mesophases often dissolve presumably because detergents are extracted from the crystal into the lipid-like environment surrounding them. In such cases, it should be tried to entirely replace LCP with an alternate viscous carrier ${ }^{24,25,26,27,29,30}$.

Modifications to the high-speed camera test can accommodate samples where the crystals are not visible within the carrier. For example, illumination can be configured to record video using polarized light microscopy. This will cause birefringent crystals to appear (as a glow), and also reveals portions of the lipid matrix that are in the birefringent lamellar phase.

Modifications aside, it is critical that the protein crystal preparation meet as much as possible the following criteria. The crystals should be optimally sized to maximize diffraction while minimizing injector clogging. The crystal density should be high enough to yield a hit-rate sufficient to collect complete data sets, but not so dense as to compromise the jet stability (a density that is too high can usually be diluted; in the case of bR, adjustment to a hit rate of $10-30 \%$ usually worked well). The lipid matrix should be brought into the cubic phase, and the crystal suspension should be homogeneous. 
TR-SFX holds major advantages over other time-resolved crystallography techniques like Laue diffraction because: radiation damage is limited due to the "diffraction before destruction" methodology, TR-SFX has a broad time resolution over many orders of magnitude and down to the femtosecond range, the small crystals allow for better photoactivation, and the photocycles of interest need not be reversible.

TR-SFX at an XFEL using the high viscosity injector has significant advantages over a liquid jet injector in terms of sample savings. Directly comparing $\mathrm{PYP}^{6}$ and $b R^{10}$, for example, indicates a reduction by the factor of 50 for the same amount of collected diffraction images. Liquid jets on the other hand have an advantage in that the flow is fast and, data collection runs at full speed (alternating dark and light frames), without regard to injector stream speed stability (or light contamination in dark frames). While the viscous jet does add new challenges, it is worth considering that liquid jet TR-SFX uses amounts of protein that may be absolutely unreasonable to produce for many biologically relevant systems.

Currently, viscous sample preparation for TR-SFX is limited by crystal compatibility with the delivery medium. Although there have been several alternative viscous carriers implemented, none have been found to work for all cases. Additionally, sample delivery with a high viscosity injector will always be subject to clogging, or slowing even with optomozation using this protocol. Another limitation of the technique is the inherent reduction in hit rate when diluting the sample to bring it to the optomised extrudability.

In the future, TR-SFX methods can be extended from protein targets with natural chromophores to those with synthetic photoswitches. Timeresolved measurements on reactions that cannot be photoactivated must rely on mixing, temperature-jump, electric-fields, or other activation technologies that have yet to be adapted to serial crystallography. A combination of these triggering technologies together with increased availability of XFEL beamtime will, over time, lay the foundation to understand the structural dynamics of protein function on an atomic level.

\section{Disclosures}

The authors declare no conflicting interests.

\section{Acknowledgments}

We acknowledge Gebhard Schertler, Rafael Abela and Chris Milne for supporting the use of high viscosity injectors at the PSI. Richard Neutze and his team are acknowledged for discussions on time-resolved crystallography and sample delivery using high viscosity injectors. For financial support, we acknowledge the Swiss National Science Foundation for grants 31003A_141235, 31003A_159558 (to J.S.) and PZ00P3_174169 (to P.N.). This project has received funding from the European Union's Horizon 2020 research and innovation program under the Marie-SklodowskaCurie grant agreement No 701646 .

1. Neutze, R., Wouts, R., van der Spoel, D., Weckert, E., Hajdu, J. Potential for biomolecular imaging with femtosecond X-ray pulses. Nature. 406 (6797), 752-7 (2000).

2. Chapman, H.N. et al. Femtosecond X-ray protein nanocrystallography. Nature. 470 (7332), 73-77 (2011).

3. Barty, A. et al. Self-terminating diffraction gates femtosecond X-ray nanocrystallography measurements. Nature photonics. 6 (December), $35-40$ (2012)

4. Aquila, A. et al. Time-resolved protein nanocrystallography using an X-ray free-electron laser. Optics express. 20 (3), $2706-16$ (2012).

5. Panneels, V. et al. Time-resolved structural studies with serial crystallography: A new light on retinal proteins. Structural Dynamics. 2 (4), $041718(2015)$

6. Tenboer, J. et al. Time-resolved serial crystallography captures high-resolution intermediates of photoactive yellow protein. Science. $\mathbf{3 4 6}$ (6214), 1242-1246 (2014).

7. Pande, K. et al. Femtosecond structural dynamics drives the trans/cis isomerization in photoactive yellow protein. Science (New York, N.Y.). 352 (6286), 725-9 (2016).

8. Barends, T.R.M. et al. Direct observation of ultrafast collective motions in CO myoglobin upon ligand dissociation. Science (New York, N.Y.). 350 (6259), 445-50 (2015).

9. Nango, E. et al. A three-dimensional movie of structural changes in bacteriorhodopsin. Science. 354 (6319), $1552-1557$ (2016).

10. Nogly, P. et al. Retinal isomerization in bacteriorhodopsin captured by a femtosecond x-ray laser. Science. 0094 (June), eaat0094 (2018).

11. Coquelle, N. et al. Chromophore twisting in the excited state of a photoswitchable fluorescent protein captured by time-resolved serial femtosecond crystallography. Nature Chemistry. 10 (1), 31-37 (2017).

12. Kupitz, C. et al. Serial time-resolved crystallography of photosystem II using a femtosecond X-ray laser. Nature. (2014).

13. Suga, M. et al. Light-induced structural changes and the site of $\mathrm{O}=\mathrm{O}$ bond formation in PSII caught by XFEL. Nature. 543 (7643), 131-135 (2017).

14. Stagno, J.R. et al. Structures of riboswitch RNA reaction states by mix-and-inject XFEL serial crystallography. Nature. 541 (7636), $242-246$ (2017).

15. Wang, D., Weierstall, U., Pollack, L., Spence, J.C.H. Liquid Mixing Jet for XFEL Study of Chemical Kinetics. Journal of synchrotron radiation. In submiss, 1364-1366 (2014).

16. Hekstra, D.R., White, K.I., Socolich, M.A., Henning, R.W., Šrajer, V., Ranganathan, R. Electric-field-stimulated protein mechanics. Nature. 540 (7633), 400-405 (2016).

17. Liu, W., Ishchenko, A., Cherezov, V. Preparation of microcrystals in lipidic cubic phase for serial femtosecond crystallography. Nature protocols. 9 (9), 2123-2134 (2014).

18. Kupitz, C., Grotjohann, I., Conrad, C.E., Roy-Chowdhury, S., Fromme, R., Fromme, P. Microcrystallization techniques for serial femtosecond crystallography using photosystem II from Thermosynechococcus elongatus as a model system. Philosophical transactions of the Royal Society of London. Series B, Biological sciences. 369 (1647) (2014). 
19. Falkner, J.C. et al. Generation of Size-Controlled, Submicrometer Protein Crystals. Chemistry of Materials. 17 (10), $2679-2686$ (2005).

20. Weierstall, U. et al. Lipidic cubic phase injector facilitates membrane protein serial femtosecond crystallography. Nature communications. $\mathbf{5}$, 3309 (2014).

21. Landau, E.M., Rosenbusch, J.P. Lipidic cubic phases: a novel concept for the crystallization of membrane proteins. Proceedings of the National Academy of Sciences of the United States of America. 93 (December), 14532-14535 (1996).

22. Caffrey, M. A comprehensive review of the lipid cubic phase or in meso method for crystallizing membrane and soluble proteins and complexes. Acta Crystallographica Section F Structural Biology Communications. 71 (1), 3-18 (2015).

23. Cherezov, V. Lipidic cubic phase technologies for membrane protein structural studies. Current Opinion in Structural Biology. 21 (4), $559-566$ (2011).

24. Conrad, C.E. et al. A novel inert crystal delivery medium for serial femtosecond crystallography. IUCrJ. 2 (4), 421-430 (2015).

25. Sugahara, M. et al. Hydroxyethyl cellulose matrix applied to serial crystallography. Scientific Reports. 7 (1), 703 (2017).

26. Sugahara, M. et al. Grease matrix as a versatile carrier of proteins for serial crystallography. Nature Methods. 12 (1), 61-63 (2014).

27. Sugahara, M. et al. Oil-free hyaluronic acid matrix for serial femtosecond crystallography. Scientific Reports. 6 (1), 24484 (2016).

28. Botha, S. et al. Room-temperature serial crystallography at synchrotron X-ray sources using slowly flowing free-standing high-viscosity microstreams. Acta Crystallographica Section D Biological Crystallography. 71 (2), 387-397 (2015).

29. Kovácsová, G. et al. Viscous hydrophilic injection matrices for serial crystallography. IUCrJ. 4 (4), $400-410$ (2017).

30. Martin-Garcia, J.M. et al. Serial millisecond crystallography of membrane and soluble protein microcrystals using synchrotron radiation. IUCrJ. 4 (4), 439-454 (2017).

31. Fromme, R. et al. Serial femtosecond crystallography of soluble proteins in lipidic cubic phase. IUCrJ. 2, 545-551 (2015).

32. Caffrey, M., Li, D., Howe, N., Shah, S.T.A. "Hit and run" serial femtosecond crystallography of a membrane kinase in the lipid cubic phase. Philosophical Transactions of the Royal Society B: Biological Sciences. 369 (1647), 20130621-20130621 (2014).

33. Liu, W. et al. Serial femtosecond crystallography of G protein-coupled receptors. Science (New York, N.Y.). 342 (6165), 1521-4 (2013).

34. Zhang, H. et al. Structure of the Angiotensin Receptor Revealed by Serial Femtosecond Crystallography. Cell. 161 (4), 833-844 (2015)

35. Fenalti, G. et al. Structural basis for bifunctional peptide recognition at human $\delta$-opioid receptor. Nature Structural \& Molecular Biology. (February) (2015).

36. Kang, Y. et al. Crystal structure of rhodopsin bound to arrestin by femtosecond X-ray laser. Nature. 523 (7562), 561-567 (2015).

37. Ishchenko, A., Cherezov, V., Liu, W. Preparation and Delivery of Protein Microcrystals in Lipidic Cubic Phase for Serial Femtosecond Crystallography. Journal of Visualized Experiments. 9 (115), 2123-34 (2016).

38. Batyuk, A. et al. Native phasing of $x$-ray free-electron laser data for a G protein-coupled receptor. Science Advances. 2 (9), e1600292e1600292 (2016).

39. Nakane, T. et al. Native sulfur/chlorine SAD phasing for serial femtosecond crystallography. Acta Crystallographica Section D Biological Crystallography. 71 (12), 2519-2525 (2015).

40. Nogly, P. et al. Lipidic cubic phase serial millisecond crystallography using synchrotron radiation. IUCrJ. 2 (2), 168-176 (2015).

41. Weinert, T. et al. Serial millisecond crystallography for routine room-temperature structure determination at synchrotrons. Nature Communications. 8 (1), 542 (2017).

42. Tosha, T. et al. Capturing an initial intermediate during the P450nor enzymatic reaction using time-resolved XFEL crystallography and cagedsubstrate. Nature Communications. 8 (1), 1585 (2017).

43. Nogly, P. et al. Lipidic cubic phase injector is a viable crystal delivery system for time-resolved serial crystallography. Nature Communications. 7, 12314 (2016).

44. Abela, R. et al. Perspective: Opportunities for ultrafast science at SwissFEL. Structural Dynamics. 4 (6), 061602 (2017).

45. Marx, V. Structural biology: doors open at the European XFEL. Nature Methods. 14 (9), 843-846 (2017).

46. Cheng, A., Hummel, B., Qiu, H., Caffrey, M. A simple mechanical mixer for small viscous lipid-containing samples. Chemistry and Physics of Lipids. 95 (1), 11-21 (1998).

47. Qiu, H., Caffrey, M. The phase diagram of the monoolein/water system: Metastability and equilibrium aspects. Biomaterials. 21 (3), $223-234$ (2000).

48. James, D. Injection Methods and Instrumentation for Serial X-ray Free Electron Laser Experiments. (2015).

49. Schindelin, J. et al. Fiji: an open-source platform for biological-image analysis. Nature Methods. 9 (7), 676-682 (2012).

50. Moffat, K. Femtosecond structural photobiology. Science (New York, N.Y.). 361 (6398), 127-128 (2018). 